\title{
Questes
}

Revue pluridisciplinaire d'études médiévales

Journée d'étude 1 - Trier, classer, organiser | 2022

Trier, classer, organiser

\section{Éclairer des mondes en peau de chèvre : intérêts des commanditaires et choix de classement des cartulaires champenois du XIII ${ }^{\mathrm{e}}$ siècle}

Thomas Lacomme

\section{(2) OpenEdition}

1 Journals

Édition électronique

URL : https://journals.openedition.org/questes/6047

DOI : 10.4000/questes. 6047

ISSN : 2109-9472

Éditeur

Les Amis de Questes

Édition imprimée

Pagination : 210-228

ISSN : 2102-7188

Référence électronique

Thomas Lacomme, «Éclairer des mondes en peau de chèvre : intérêts des commanditaires et choix de classement des cartulaires champenois du xIII siècle », Questes [En ligne], Journée d'étude 1 - Trier, classer, organiser | 2022, mis en ligne le 31 janvier 2022, consulté le 21 février 2022. URL : http:// journals.openedition.org/questes/6047 ; DOI : https://doi.org/10.4000/questes.6047 


\title{
Éclairer des mondes en peau de chèvre : intérêts des commanditaires et choix de classement des cartulaires champenois du $\mathrm{XIII}^{\mathrm{e}}$ siècle
}

\author{
Thomas LACOMME \\ École Pratique des Hautes Études
}

Le tri, processus qui allie conservation et élimination, est à l'œuvre dans toute cartularisation. Les cartulaires ne sont pas les reflets des chartriers ${ }^{1}$ : pourquoi certains actes furent-ils copiés dans le cartulaire alors que d'autres ne le furent pas ? Comment et dans quel but la sélection a-t-elle été effectuée ? Le renouveau historiographique de l'étude des cartulaires et des écrits de gestion encourage à s'interroger sur ces choix ${ }^{2}$. Souci de conservation, construction mémorielle, rationalisation des opérations de gestion, nécessités juridiques ou politiques: les raisons qui président à la constitution d'un cartulaire sont nombreuses. Pour comprendre ce qui pousse une institution ou une personne

\footnotetext{
${ }^{1}$ Un cartulaire correspond à «toute transcription organisée (sélective ou exhaustive) de documents diplomatiques, réalisée par le détenteur de ceux-ci ou pour son compte, afin d'en assurer la conservation et d'en faciliter la consultation » (Olivier Guyotjeannin, Jacques Pycke et Benoît-Michel Tock, Diplomatique médiévale, Turnhout, Brepols, 1993, p. 277). Un chartrier est au sens strict «l'ensemble des chartes conservées par une personne physique ou morale - le plus souvent, un seigneur, une institution ecclésiastique, une ville - pour faire la preuve de ses droits ou conserver la mémoire de son histoire » (Vocabulaire international de la diplomatique, dir. Maria Milagros Carcel Orti, Valence, Université de Valence, 1997 [ 2 édition corrigée], p. 27).

${ }^{2}$ Sur les cartulaires, voir Les Cartulaires. Actes de la table ronde organisée par l'École nationale des chartes et le GDR 121 du CNRS les 5 et 7 décembre 1991, dir. Olivier Guyotjeannin, Laurent Morelle et Michel Parisse, Paris, École des chartes, 1993 ; Pierre Chastang, Lire, écrire, transcrire. Le travail des rédacteurs de cartulaires en Bas-Languedoc (XI $I^{e}$-XIII siècles), Paris, CTHS, 2001. Sur les écrits de gestion, Décrire, inventorier, enregistrer entre Seine et Rhin au Moyen Âge: formes, fonctions et usages des écrits de gestion. Actes du colloque international organisé à l'université de Namur (FUNDP) les 8 et 9 mai 2008, dir. voir Xavier Hermand, Jean-François Nieus et Étienne Renard, Paris, École des chartes, 2012. 210
} 
privée à faire transcrire la totalité ou une partie des documents diplomatiques qu'elle conserve, il faut donc analyser le contexte et les intérêts particuliers des commanditaires.

Le classement du matériau diplomatique issu de ce processus de tri est une opération qui suppose, elle aussi, un certain nombre de choix. Au sein d'une même région, à une époque donnée, les cartulaires ne semblent en effet pas obéir aux mêmes logiques de classement, malgré les similitudes du matériau collecté. Leurs classements peuvent être topographiques, hiérarchiques, thématiques, rarement chronologiques. Ils sont souvent mixtes parce que les logiques de classement peuvent se cumuler et entrer ainsi en complémentarité ou en concurrence. Plusieurs campagnes de cartularisation peuvent avoir eu lieu et des changements d'ordre à l'intérieur des cartulaires ont pu intervenir, parasitant les classements originels. Il faut aussi envisager le caractère lent ou progressif de la systématisation des classements ${ }^{3}$.

Les scribes sont humains, et l'erreur, l'oubli, des problèmes matériels, la possibilité de l'abandon d'un choix de classement, sont autant de situations qui doivent être envisagées pour comprendre que, si le cartulaire est organisé, il est aussi, en un sens, organique. Étudier des cartulaires ne doit donc pas consister à produire un discours normatif, le risque étant d'édifier un système qui mépriserait les ruptures ou qualifierait d'anomalies des éléments qui pourraient bien être la trace de l'évolution du document ou le révélateur des logiques multiples et parfois concurrentes qui ont présidé à son organisation. Les paratextes des cartulaires, notamment les préfaces, les tables des actes ou les rubriques, peuvent enfin nous renseigner sur les choix des cartularistes.

Le regroupement de certains actes jugés semblables donne lieu à des phénomènes de concentration au sein des cartulaires. Ces groupes d'actes sont eux-mêmes hiérarchisés. Les regroupements ne sont pas identiques, les

\footnotetext{
${ }^{3}$ Isabelle Rosé évoque pour la Bourgogne du XIII siècle une «généralisation du mode de classement systématique » (Isabelle Rosé, « Panorama de l'écrit diplomatique en Bourgogne : autour des cartulaires (XI ${ }^{\mathrm{e}}-\mathrm{XVIII}{ }^{\mathrm{e}}$ siècles) », Bulletin du centre d'études médiévales d'Auxerre [En ligne], 11, 2007, § 55).
} 
hiérarchies sont souvent différentes. À quelles représentations du monde ces organisations dissemblables renvoient-elles ? Paul Bertrand et Xavier Hélary ont par exemple montré que la dimention spatiale échappait souvent à la définition donnée aux cartulaires ${ }^{4}$.

En ordonnant les actes, les cartularistes ordonnent le monde ou, du moins, dévoilent une représentation de la société qui les entoure et qui parle à travers les chartes émises et collectées. Les cartulaires ne sont pas des cartes, pourtant ils délimitent les contours de mondes particuliers à leurs commanditaires et le développement qui suit entend l'illustrer, à partir d'exemples tirés de la Champagne méridionale du XIII ${ }^{\mathrm{e}}$ siècle. Notre étude commencera par une comparaison entre deux cartulaires issus de deux établissements religieux, celui de la collégiale séculière Saint-Étienne de Troyes $^{5}$, fondée avant 1157 , et celui d'une abbaye sise en la même ville depuis le VII ${ }^{\mathrm{e}}$ siècle, Saint-Loup ${ }^{6}$, deux cartulaires qui obéissent à un classement hiérarchique. Nous retrouverons cette influence des intérêts des commanditaires sur les classements des actes, d'abord en comparant le cartulaire de la comtesse Blanche et les cartulaires de chancellerie, ensuite en examinant le cartulaire du marchand italien Renier Accore.

\footnotetext{
${ }^{4}$ Paul Bertrand et Xavier Hélary, «Constructions de l'espace dans les cartulaires », dans Construction de l'espace au Moyen Âge: pratiques et représentations. XXXVII Congrès de la SHMES, Société des historiens médiévistes de l'enseignement supérieur public, Mulhouse, 2-4 juin 2006, dir. Thomas Lienhard, Paris, Publications de la Sorbonne, 2007, p. 193-207. Voir aussi Florian Mazel, «L'espace du diocèse dans les cartulaires cathédraux (fin du XI ${ }^{\mathrm{e}}-$ début du $\mathrm{XIV}^{\mathrm{e}}$ siècle) », dans L'Espace du diocèse : genèse d'un territoire dans l'Occident médiéval, $V^{e}$-XIII siècle, dir. Florian Mazel, Rennes, Presses Universitaires de Rennes, 2008, p. 367-400.

${ }^{5}$ Paris, BnF, ms. lat. 17098 (années 1270, pour la première campagne de cartularisation), 394 fol. L'édition du cartulaire, en grande partie inédit, est l'un des enjeux de ma thèse, dirigée par M. Laurent Morelle à l'École Pratique des Hautes Études. Sur le cartulaire de la collégiale Saint-Étienne de Troyes, voir Thomas Lacomme, «Un cartulaire sous influence? Edmond de Lancastre, Jean d'Acre, Renier Acorre et le cartulaire de la collégiale séculière Saint-Étienne de Troyes » dans Aux sources du pouvoir. Voir, approcher, comprendre le pouvoir politique au Moyen Âge, dir. Sylvain Gouguenheim, Paris, Les Indes Savantes, 2017, p. 87-110.

${ }^{6}$ Troyes, BM, ms. 2755 ( $2^{\mathrm{e}}$ moitié du XIII ${ }^{\mathrm{e}}$ siècle), 90 fol. Pour une édition du cartulaire de Saint-Loup, voir Charles Lalore, Collection des principaux cartulaires du diocèse de Troyes, t. I, Cartulaire de l'abbaye Saint-Loup de Troyel2, Paris/Troyes, Thorin/Lacroix, 1875.
} 


\section{Comparaison et modélisation graphique des cartulaires de Saint- Étienne et de Saint-Loup de Troyes}

Sur la dizaine de cartulaires d'établissements religieux produits en Champagne au XIII ${ }^{\mathrm{e}}$ siècle, ceux de Saint-Étienne et de Saint-Loup de Troyes, ainsi que celui de l'abbaye Notre-Dame de Larrivour ${ }^{7}$, sont les seuls dont les classements peuvent être comparés sans crainte, parce qu'ils regroupent, tous les trois, un nombre suffisant d'actes et parce qu'ils sont conservés en originaux, de manière complète et intacte. Ces critères méthodologiques ont conduit à ne pas prendre en considération un certain nombre de cartulaires ecclésiastiques produits en Champagne à la même époque ${ }^{8}$. Sur les trois cartulaires retenus, ceux de Saint-Étienne et de Saint-Loup affichent le même type de classement, globalement hiérarchique, alors que celui de Notre-Dame de Larrivour présente un classement topographique.

Pour comprendre le classement hiérarchique des deux premiers cartulaires, j'ai regroupé les auteurs d'actes par catégories: comtes de Champagne; comtesses de Champagne ; vassaux et agents des comtes; autres acteurs laïques ; papes (ou patriarches ${ }^{9}$ ) ; archevêques et évêques ; archidiacres, doyens de chrétienté et officiaux ; chapitres cathédraux ; collégiales ; abbayes et prieurés; autres acteurs religieux. J'ai aussi pris en compte les paratextes, les

\footnotetext{
${ }^{7}$ Troyes, AD Aube, 4 H 1 (XIII ${ }^{\mathrm{e}}$ siècle), 98 fol.

${ }^{8}$ Parce qu'ils ne répondaient pas aux critères évoqués, les cartulaires suivants ont été écartés : celui du chapitre cathédral Saint-Pierre de Troyes, parce qu'il n'est pas conservé en original et qu'il est connu seulement par la reconstitution qu'en a donnée un érudit champenois, pour un résultat d'ailleurs très contestable (Charles Lalore, Collection des principaux cartulaires $d u$ diocèse de Troyes, t. V, Cartulaire de Saint-Pierre de Troyes; chartes de la collégiale de Saint-Urbain de Troyes, Paris/Troyes, Thorin/Lacroix, 1880) ; ceux des abbayes de NotreDame du Reclus (Châlons-en-Champagne, AD Marne, H, non côté) et de Notre-Dame de la Chapelle-aux-Planches (Choignes, AD Haute-Marne, $4 \mathrm{H} \mathrm{1)}$, parce qu'avec respectivement 18 et 30 folios ils ne contiennent pas assez d'actes; celui de l'abbaye Saint-Pierre de Montiéramey (Troyes, AD Aube, 6 H 25), parce qu'il est incomplet ; celui de l'abbaye NotreDame de Scellières (Troyes, BM, ms. 2290), parce qu'il a été mutilé.

${ }^{9} \mathrm{Au}$ folio $56 \mathrm{du}$ cartulaire de Saint-Loup, on trouve en effet un acte du patriarche de Constantinople, Gervais, datant de 1216, pris à Corinthe le 29 mai. Il confirme la concession faite par Eudes, évêque de Coron, à l'abbaye de Saint-Loup de Troyes de l'abbaye du SaintSauveur dans le diocèse de Modon (Medone), en Grèce. Voir Jean Longnon, « Le patriarcat latin de Constantinople », Journal des savants, 4, 1941, p. 174-184.
} 
documents non diplomatiques et les folios laissés blancs, afin d'étuder

l'ensemble de l'espace codicologique des deux cartulaires. J'ai ensuite réalisé des tableaux, où j'ai reporté les regroupements d'actes par folios et les catégories d'auteurs (tableaux 1 et 2).

\section{Tableau 1}

\section{L'organisation du cartulaire de Saint-Étienne de Troyes}

\section{par catégories d'auteurs d'actes}

\begin{tabular}{|c|c|}
\hline Folios & Catégories d'auteurs d'acte \\
\hline $1-30$ & Paratexte \\
\hline $31-53$ & Comtes de Champagne \\
\hline 54 & Folio blanc \\
\hline $55 \mathrm{ra}$ & Vassaux et agents des comtes \\
\hline $55 \mathrm{va}-66 \mathrm{ra}$ & Comtesses de Champagne \\
\hline $66 \mathrm{rb}-72 \mathrm{vb}$ & Vassaux et agents des comtes \\
\hline $72 \mathrm{vb}-73 \mathrm{ra}$ & Autres auteurs laïques \\
\hline $73 \mathrm{rb}-75 \mathrm{rb}$ & Vassaux et agents des comtes \\
\hline $75 \mathrm{va}-76 \mathrm{rb}$ & Autres auteurs laïques \\
\hline $76 v a-81 v a$ & Vassaux et agents des comtes \\
\hline $81 \mathrm{vb}-82 \mathrm{ra}$ & Autres auteurs laïques \\
\hline $82 \mathrm{rb}-88 \mathrm{va}$ & Vassaux et agents des comtes \\
\hline 89ra - 103ra & Papes \\
\hline $103 \mathrm{rb}$ & Texte non diplomatique \\
\hline $104 \mathrm{ra}-115 \mathrm{va}$ & Archevêques et évêques \\
\hline $116 \mathrm{ra}-126 \mathrm{rb}$ & Chapitres cathédraux \\
\hline 127 & Folio blanc \\
\hline $128 \mathrm{va}-141 \mathrm{ra}$ & Collégiales \\
\hline $141 \mathrm{rb}-142 \mathrm{ra}$ & $\begin{array}{l}\text { Archidiacres, doyens } \\
\text { et officiaux }\end{array}$ \\
\hline $142 \mathrm{ra}-146 \mathrm{vb}$ & Collégiales \\
\hline $146 \mathrm{vb}-147 \mathrm{rb}$ & $\begin{array}{l}\text { Archidiacres, doyens } \\
\text { et officiaux }\end{array}$ \\
\hline $147 \mathrm{va}-148 \mathrm{va}$ & Collégiales \\
\hline $148 \mathrm{va}$ & $\begin{array}{l}\text { Archidiacres, doyens et } \\
\text { officiaux }\end{array}$ \\
\hline 149ra - 150ra & Collégiales \\
\hline $150 \mathrm{va}-151 \mathrm{rb}$ & $\begin{array}{l}\text { Archidiacres, doyens et } \\
\text { officiaux }\end{array}$ \\
\hline $151 \mathrm{rb}-\mathrm{vb}$ & Autres auteurs ecclésiastiques \\
\hline $152 \mathrm{ra}-\mathrm{b}$ & Collégiales \\
\hline $152 \mathrm{va}$ & Autres auteurs ecclésiastiques \\
\hline $152 \mathrm{vb}-153 \mathrm{ra}$ & Collégiales \\
\hline $153 \mathrm{va}-154 \mathrm{ra}$ & Autres auteurs ecclésiastiques \\
\hline $154 \mathrm{rb}-\mathrm{vb}$ & $\begin{array}{l}\text { Archidiacres, doyens et } \\
\text { officiaux }\end{array}$ \\
\hline $155 \mathrm{ra}-156 \mathrm{va}$ & Collégiales \\
\hline $156 \mathrm{vb}$ & Chapitres cathédraux \\
\hline $157 \mathrm{ra}-\mathrm{va}$ & Autres auteurs ecclésiastiques \\
\hline $157 \mathrm{vb}-158 \mathrm{rb}$ & Collégiales \\
\hline $158 \mathrm{rb}-\mathrm{va}$ & Chapitres cathédraux \\
\hline $158 \mathrm{va}-159 \mathrm{vb}$ & Collégiales \\
\hline
\end{tabular}

\begin{tabular}{|c|c|}
\hline 160 & Abbayes et prieurés \\
\hline 161 & Folio blanc \\
\hline $162 \mathrm{ra}-\mathrm{vb}$ & Abbayes et prieurés \\
\hline $162 \mathrm{vb}-163 \mathrm{ra}$ & Autres auteurs laïques \\
\hline $163 \mathrm{ra}-173 \mathrm{rb}$ & Abbayes et prieurés \\
\hline $173 \mathrm{rb}-\mathrm{va}$ & Collégiales \\
\hline $174-175$ & Folios blancs \\
\hline 176ra-b & Abbayes et prieurés \\
\hline $176 \mathrm{rb}-\mathrm{vb}$ & Collégiales \\
\hline $177 \mathrm{ra}-182 \mathrm{rb}$ & Abbayes et prieurés \\
\hline 183 & Folio blanc \\
\hline $184 \mathrm{ra}-188 \mathrm{rb}$ & Abbayes et prieurés \\
\hline 189 & Folio blanc \\
\hline 190ra-191rb & Abbayes et prieurés \\
\hline $191 v a-b$ & Vassaux et agents des comtes \\
\hline 192 & Abbayes et prieurés \\
\hline 193 & Folio blanc \\
\hline $194 \mathrm{ra}-200 \mathrm{va}$ & Abbayes et prieurés \\
\hline $200 v a-b$ & Vassaux et agents des comtes \\
\hline 201 & Folio blanc \\
\hline 202ra - 206va & $\begin{array}{l}\text { Archidiacres, doyens et } \\
\text { officiaux }\end{array}$ \\
\hline $207-209$ & Folios blancs \\
\hline $210 \mathrm{ra}-234 \mathrm{vb}$ & $\begin{array}{l}\text { Archidiacres, doyens et } \\
\text { officiaux }\end{array}$ \\
\hline $235 \mathrm{ra}$ & Autres auteurs laïques \\
\hline $235 \mathrm{ra}-310 \mathrm{rb}$ & $\begin{array}{l}\text { Archidiacres, doyens et } \\
\text { officiaux }\end{array}$ \\
\hline $310 \mathrm{rb}-311 \mathrm{ra}$ & Autres auteurs ecclésiastiques \\
\hline $311 \mathrm{rb}-312 \mathrm{va}$ & Collégiales \\
\hline $312 v b-333 v a$ & $\begin{array}{l}\text { Archidiacres, doyens et } \\
\text { officiaux }\end{array}$ \\
\hline $333 \mathrm{vb}-335 \mathrm{rb}$ & Vassaux et agents des comtes \\
\hline 336 & Folio blanc \\
\hline $337 \mathrm{ra}-\mathrm{va}$ & Comtes de Champagne \\
\hline $337 \mathrm{vb}$ & Comtesses de Champagne \\
\hline 338ra - 339ra & Comtes de Champagne \\
\hline 340ra-b & Comtesses de Champagne \\
\hline $340 \mathrm{rb}-\mathrm{vb}$ & Comtes de Champagne \\
\hline $341 \mathrm{ra}-\mathrm{b}$ & $\begin{array}{l}\text { Archidiacres, doyens et } \\
\text { officiaux }\end{array}$ \\
\hline $341 \mathrm{rb}-\mathrm{va}$ & Abbayes et prieurés \\
\hline $341 v a-342 \mathrm{rb}$ & Archidiacres, doyens et \\
\hline
\end{tabular}




\begin{tabular}{|l|l|}
\hline & officiaux \\
\hline 342va - 343ra & Comtes de Champagne \\
\hline 343rb - va & Vassaux et agents des comtes \\
\hline 343vb - 344vb & $\begin{array}{l}\text { Archidiacres, doyens et } \\
\text { officiaux }\end{array}$ \\
\hline 345ra - 348ra & Vassaux et agents des comtes \\
\hline 348ra-b & Collégiales \\
\hline 348va - 364vb & Vassaux et agents des comtes \\
\hline 364vb - 365rb & Autres auteurs laïques \\
\hline 365rb - 388ra & Vassaux et agents des comtes \\
\hline
\end{tabular}

\begin{tabular}{|l|l|}
\hline 389ra $-390 \mathrm{rb}$ & Autres auteurs ecclésiastiques \\
\hline $391 \mathrm{va}-392 \mathrm{va}$ & Collégiales \\
\hline 393 & Folio blanc \\
\hline 394 & Vassaux et agents des comtes \\
\hline
\end{tabular}

\section{Tableau 2}

\section{L'organisation du cartulaire de Saint-Loup de Troyes \\ par catégories d'auteurs d'actes}

\begin{tabular}{|c|c|}
\hline Folios & Catégories d'auteurs d'acte \\
\hline $1-3$ & Paratexte \\
\hline 4 & Folio blanc \\
\hline $5-17$ & Papes \\
\hline 18 & Folio blanc \\
\hline $19 \mathrm{r}$ & Autres auteurs laïques \\
\hline $19 \mathrm{v}$ & Comtes de Champagne \\
\hline 20 & Folio blanc \\
\hline $21-22$ & Archevêques et évêques \\
\hline $23 r-v$ & Vassaux et agents des comtes \\
\hline $23 v-25 v$ & Archevêques et évêques \\
\hline $25 \mathrm{v}$ & Abbayes et prieurés \\
\hline $26-36 v$ & Archevêques et évêques \\
\hline $37 \mathrm{r}$ & Chapitres cathédraux \\
\hline $37 \mathrm{r}$ & Vassaux et agents des comtes \\
\hline $37 \mathrm{v}-41 \mathrm{r}$ & Comtes de Champagne \\
\hline $41 \mathrm{r}$ & Vassaux et agents des comtes \\
\hline $41 r-43 v$ & Comtes de Champagne \\
\hline $43 v-46 r$ & Comtesses de Champagne \\
\hline $46 r-52 v$ & Vassaux et agents des comtes \\
\hline $52 \mathrm{v}$ & $\begin{array}{l}\text { Archidiacres, doyens et } \\
\text { officiaux }\end{array}$ \\
\hline $52 \mathrm{v}$ & Vassaux et agents des comtes \\
\hline $52 \mathrm{v}$ & $\begin{array}{l}\text { Archidiacres, doyens et } \\
\text { officiaux }\end{array}$ \\
\hline 53 & Comtes de Champagne \\
\hline $53 v-54 r$ & Vassaux et agents des comtes \\
\hline $54 v$ & Comtesses de Champagne \\
\hline $54 v-56 r$ & Vassaux et agents des comtes \\
\hline $56 \mathrm{r}-\mathrm{v}$ & Patriarche de Constantinople \\
\hline $56 v-57 v$ & Archevêques et évêques \\
\hline $57 v-63 r$ & $\begin{array}{l}\text { Archidiacres, doyens et } \\
\text { officiaux }\end{array}$ \\
\hline $63 \mathrm{r}$ & Autres auteurs ecclésiastiques \\
\hline $63 r-v$ & Abbayes et prieurés \\
\hline 64 & Chapitres cathédraux \\
\hline $64 v-65 v$ & Abbayes et prieurés \\
\hline $65 v-66 r$ & Collégiales \\
\hline $66 r$ & Abbayes et prieurés \\
\hline $66 r-v$ & Archidiacres, doyens et \\
\hline
\end{tabular}

\begin{tabular}{|c|c|}
\hline & officiaux \\
\hline $66 v$ & Abbayes et prieurés \\
\hline $66 v-67 r$ & Vassaux et agents des comtes \\
\hline $67 v$ & Papes \\
\hline $68-69 r$ & Chapitres cathédraux \\
\hline $69 r-71 v$ & Collégiales \\
\hline $71 v-73 r$ & Abbayes et prieurés \\
\hline $73 r-v$ & $\begin{array}{l}\text { Archidiacres, doyens et } \\
\text { officiaux }\end{array}$ \\
\hline $73 v-74 r$ & Abbayes et prieurés \\
\hline $74 r-v$ & $\begin{array}{l}\text { Archidiacres, doyens et } \\
\text { officiaux }\end{array}$ \\
\hline $74 \mathrm{v}$ & Abbayes et prieurés \\
\hline 75 & Folio blanc \\
\hline 76 & Papes \\
\hline $77 \mathrm{r}$ & Comtesses de Champagne \\
\hline $77 \mathrm{r}-\mathrm{v}$ & $\begin{array}{l}\text { Archidiacres, doyens et } \\
\text { officiaux }\end{array}$ \\
\hline $77 \mathrm{v}-78 \mathrm{r}$ & Archevêques et évêques \\
\hline $78 \mathrm{r}$ & $\begin{array}{l}\text { Archidiacres, doyens et } \\
\text { officiaux }\end{array}$ \\
\hline $78 \mathrm{r}$ & Archevêques et évêques \\
\hline $78 r-v$ & $\begin{array}{l}\text { Archidiacres, doyens et } \\
\text { officiaux }\end{array}$ \\
\hline $78 v$ & Archevêques et évêques \\
\hline $78 v-79 r$ & $\begin{array}{l}\text { Archidiacres, doyens et } \\
\text { officiaux }\end{array}$ \\
\hline $79 \mathrm{r}-\mathrm{v}$ & Vassaux et agents des comtes \\
\hline $79 v-80 r$ & Comtesses de Champagne \\
\hline $80 r-v$ & $\begin{array}{l}\text { Archidiacres, doyens et } \\
\text { officiaux }\end{array}$ \\
\hline $80 v-81 r$ & Collégiales \\
\hline $81 \mathrm{r}$ & Vassaux et agents des comtes \\
\hline $81 \mathrm{r}-\mathrm{v}$ & $\begin{array}{l}\text { Archidiacres, doyens et } \\
\text { officiaux }\end{array}$ \\
\hline $81 \mathrm{v}$ & Autres auteurs laïques \\
\hline $81 v$ & $\begin{array}{l}\text { Archidiacres, doyens et } \\
\text { officiaux }\end{array}$ \\
\hline $82 \mathrm{r}$ & Abbayes et prieurés \\
\hline $82 \mathrm{r}$ & Comtesses de Champagne \\
\hline
\end{tabular}




\begin{tabular}{|l|l|}
\hline $82 \mathrm{r}$ & Vassaux et agents des comtes \\
\hline $82 \mathrm{v}$ & $\begin{array}{l}\text { Archidiacres, doyens et } \\
\text { officiaux }\end{array}$ \\
\hline $82 \mathrm{v}-83 \mathrm{r}$ & Vassaux et agents des comtes \\
\hline $83 \mathrm{r}-84$ & Documents non diplomatiques \\
\hline $85 \mathrm{r}$ & $\begin{array}{l}\text { Archidiacres, doyens et } \\
\text { officiaux }\end{array}$ \\
\hline $85 \mathrm{r}$ & Vassaux et agents des comtes \\
\hline $85 \mathrm{r}-\mathrm{v}$ & $\begin{array}{l}\text { Archidiacres, doyens et } \\
\text { officiaux }\end{array}$ \\
\hline $85 \mathrm{v}-86$ & Vassaux et agents des comtes \\
\hline $86 \mathrm{v}$ & $\begin{array}{l}\text { Archidiacres, doyens et } \\
\text { officiaux }\end{array}$ \\
\hline $86 \mathrm{v}-87 \mathrm{r}$ & Comtesses de Champagne \\
\hline $87 \mathrm{r}$ & Vassaux et agents des comtes \\
\hline $87 \mathrm{r}-\mathrm{v}$ & Abbayes et prieurés \\
\hline
\end{tabular}

\begin{tabular}{|l|l|}
\hline $87 \mathrm{v}$ & Vassaux et agents des comtes \\
\hline $87 \mathrm{v}$ & Collégiales \\
\hline $87 \mathrm{v}-88 \mathrm{r}$ & Abbayes et prieurés \\
\hline $88 \mathrm{r}$ & Vassaux et agents des comtes \\
\hline $88 \mathrm{v}$ & Comtes de Champagne \\
\hline $89 \mathrm{r}$ & Rois de France avant 1314 \\
\hline $89 \mathrm{v}$ & Abbayes et prieurés \\
\hline $90 \mathrm{r}$ & Documents non diplomatiques \\
\hline $90 \mathrm{v}$ & Comtes de Champagne \\
\hline $90 \mathrm{v}$ & Documents non diplomatiques \\
\hline $91 \mathrm{r}-\mathrm{v}$ & Archevêques et évêques \\
\hline $91 \mathrm{v}-92 \mathrm{v}$ & Abbayes et prieurés \\
\hline $92 \mathrm{v}-94 \mathrm{v}$ & $\begin{array}{l}\text { Archidiacres, doyens et } \\
\text { officiaux }\end{array}$ \\
\hline $94 \mathrm{v}$ & Documents non diplomatiques \\
\hline
\end{tabular}


Comme une représentation sous la forme d'un tableau lisse les phénomènes et écrase leur échelle, pour représenter au mieux l'espace codicologique, je propose de le modéliser sous forme de frise, en indiquant par des couleurs ${ }^{10}$ les différentes catégories d'auteurs d'acte (figs. 1 et 2). L'idée d'une telle modélisation découle de la lecture de la méthode, proposée par Julio Escalona, Cristina Jular Perez-Alfaro et Anna Bellettini, pour réaliser une modélisation de l'organisation chronologique des cartulaires ${ }^{11}$. Pour ma part, je propose une modélisation de l'organisation hiérarchique des cartulaires.

L'examen des modélisations graphiques du cartulaire de SaintÉtienne et de Saint-Loup montre que les séries hiérarchiques sont rarement parfaites : les ajouts postérieurs et les nouvelles campagnes de cartularisation en sont en grande partie responsables. Le cartulaire de Saint-Loup, par exemple, affiche des séries globalement cohérentes, qui se succèdent les unes les autres, malgré quelques accidents, jusqu'au folio 75 laissé blanc. Il s'agit d'une sorte de frontière dans l'espace codicologique, qui détermine deux mondes : avant ce folio, les actes ont

\footnotetext{
${ }^{10}$ Le choix des couleurs reprend la division entre auteurs laïcs (couleurs froides) et auteurs ecclésiastiques (couleurs chaudes). Si la répartition laïcs-couleurs froides/ecclésiastiques-couleurs chaudes a pu être guidée par la référence culturelle de la pourpre cardinalice, des raisons d'ordre pratique expliquent aussi ce choix : pour faire figurer des sous-catégories plus nombreuses du côté du monde ecclésiastiques, la palette des couleurs chaudes semblait sinon plus fournie, du moins offrir plus facilement des nuances rapidement repérables et lisibles. Dans le détail, on notera que le violet des collégiales, dans lequel se fondent les actes de la collégiale séculière Saint-Étienne de Troyes, est un mélange de rouge et de bleu, cette collégiale étant un établissement religieux fondé par un laïc. Pour une sémiologie des couleurs et de leur utilisation dans les graphiques, voir Jean Bertin, Sémiologie graphique: les diagrammes, les réseaux, les cartes, Paris/La Haye, Gauthier-Villard/Mouton, 1967 ; George H. Joblove et Donald Greenberg, «Color spaces for computer graphics », ACM Siggraph Computer Graphics, vol. 12, n 3, p. 20-25 ; Sidonie Christophe, Aide à la conception de légendes personnalisées et originales : proposition d'une méthode coopérative pour le choix des couleurs, thèse de sciences de l'information géographique sous la dir. d'Anne Ruas, Université Paris-Est, 2009.

${ }^{11}$ Julio Escalona, Cristina Jular Perez-Alfaro et Anna Bellettini, «Two graphical models for the analysis and comparison of cartularies », Digital Medievalist, 10, 2016-2017 [En ligne].
} 
majoritairement été transcrits par la même main et respectent une même logique, hiérarchique, de classement. La majorité des actes issus d'une seconde campagne de cartularisation ou qui correspondent à des ajouts ponctuels ont été transcrits à partir du folio 76 et jusqu'au folio 94, sans logique apparente de classement, la succession rapide des bandes colorées de la modélisation graphique rendant bien compte de ce côté moins ordonné de la fin du cartulaire de Saint-Loup.

Les modélisations proposées permettent d'étudier l'organisation des cartulaires tels qu'ils se présentent aujourd'hui. Pour essayer de rendre compte du classement originel, de son parasitage par les ajouts postérieurs et de l'évolution des cartulaires, il faudrait pouvoir prendre en compte le facteur temporel dans la modélisation graphique. Pour cela, il serait possible de réaliser plusieurs frises, qui obéiraient à la méthode que nous venons d'exposer : la première frise correspondrait à l'organisation originelle des cartulaires, la ou les suivantes aux campagnes de cartularisation postérieures ou aux rajouts. Une autre option consisterait à ne réaliser qu'une seule frise, qui correspondrait à l'état final du cartulaire, mais sur laquelle il faudrait indiquer, par un figuré différent des applats de couleurs, les étapes de l'évolution du cartulaire. 

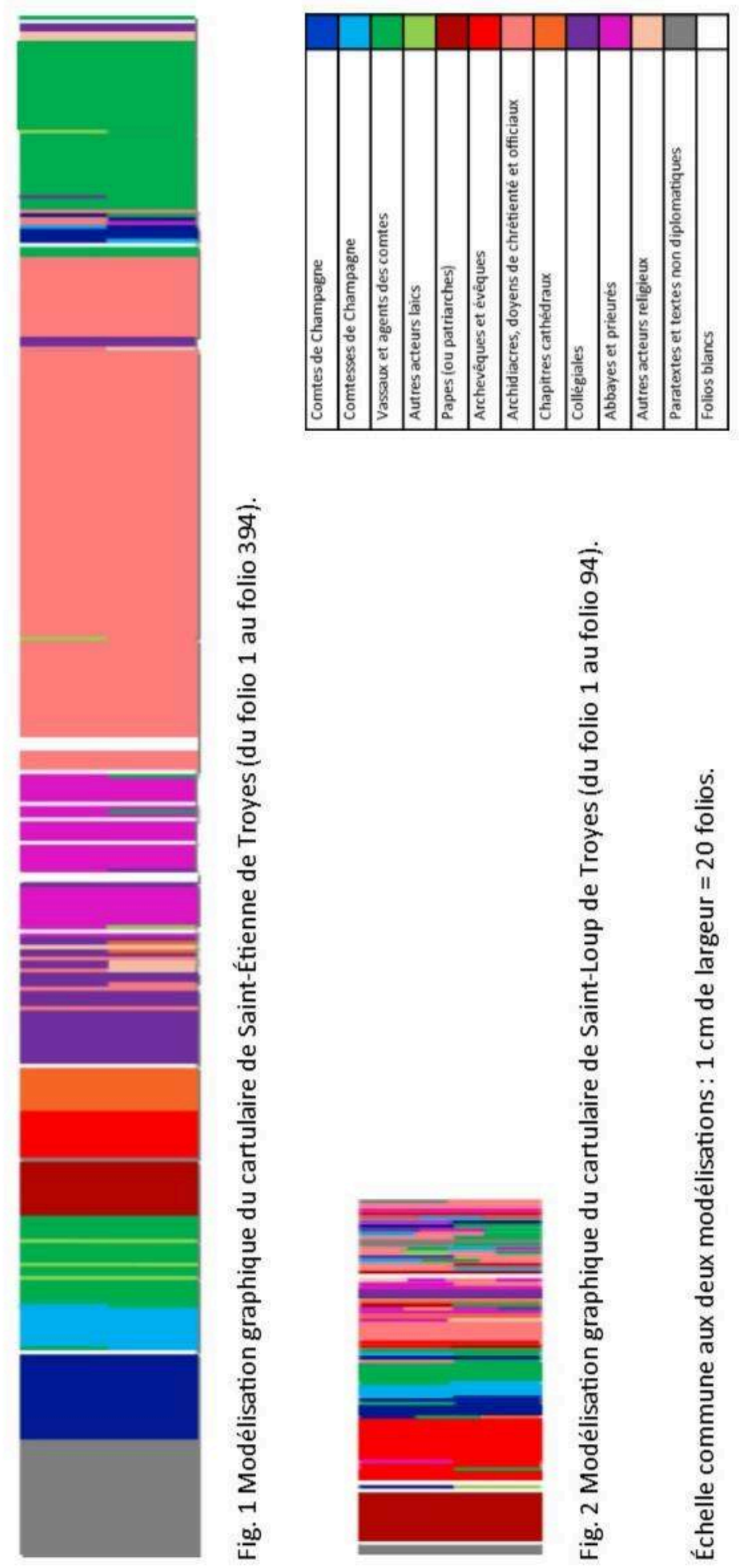

Modélisations graphiques des cartulaires de Saint-Étienne et de Saint-Loup de Troyes 


\section{Le comte avant l'évêque : le classement des actes peut-il refléter le contexte politique?}

Les modélisations graphiques des deux cartulaires champenois prouvent qu'une même logique de classement, ici hiérarchique, peut aboutir à des organisations internes différentes, entre deux cartulaires produits à une même époque dans une même région. Le cartulaire de Saint-Loup commence par un paratexte ${ }^{12}$, puis viennent les privilèges pontificaux, séparés du paratexte et du reste du cartulaire par deux folios laissés blancs ${ }^{13}$ (fol. 4 et fol. 18). Après les papes, conformément à la hiérarchie de l'Église, viennent les évêques, puis le cartulaire change de monde et présente des actes des comtes, puis des comtesses, puis des vassaux et agents comtaux, avant de revenir dans le monde ecclésiastique en présentant d'abord des actes du chapitre cathédral puis ceux des archidiacres, doyens de chrétienté et officiaux, etc. Cette hiérarchie semble attendue dans le contexte post-grégorien, mais aussi dans celui de la division du pouvoir épiscopal et de l'autonomisation, d'une part du chapitre cathédral, d'autre part des autorités recevant délégation du pouvoir épiscopal : archidiacres, doyens de chrétientés, officiaux ${ }^{14}$.

\footnotetext{
12 Il s'agit des mémoires de l'abbé Guitère, datées d'avant 1181, dont Charles Lalore a reconstitué la tradition, qui n'est pas simple : les folios sur lesquels elles étaient copiées ne figurent plus dans le ms. 2755 mais en ont été détachés pour être insérés dans le ms. 2275 ad calcem de la BM de Troyes, qui est un évangéliaire donné à Saint-Loup par le comte Henri le Libéral : Charles Lalore, Cartulaires, op. cit., t. I, p. VI (édition : p. 1-9).

${ }^{13}$ Dans l'organisation originelle du cartulaire, il y avait d'ailleurs trois folios laissés vierges entre les actes émanant de la chancellerie pontificale et les autres (fol. 18-20), les actes copiés au folio 19 étant des ajouts postérieurs à la première campagne de cartularisation. Ces folios vierges étaient-ils destinés à recevoir d'autres privilèges pontificaux, ce qui témoignerait d'une prudence des cartularistes et des espoirs de l'institution monastique troyenne ? Ne faut-il pas plutôt y lire la volonté de mettre en exergue les documents jugés les plus précieux et importants?

${ }^{14}$ Sur l'évolution des hiérarchies ecclésiastiques en contexte post-grégorien et sur les délégations du pouvoir épiscopal, voir Roger Berger, "Archidiacres, officiaux, dignitaires du chapitre d'Arras (1093-1320) Essai de chronologie », Bulletin de la
} 
A contrario, la hiérarchie des actes du cartulaire de Saint-Étienne de Troyes peut surprendre. Autant il n'est pas étonnant que le cartulaire d'une collégiale séculière fondée par un comte de Champagne commence, sinon par l'acte de fondation, au moins, comme c'est le cas ici, par l'acte de première dotation du tempore ${ }^{15}$, autant la longue série hiérarchique des actes pris par des autorités laïques a de quoi étonner, parce qu'elle semble reléguer le premier privilège pontifical au folio 89 . Après les privilèges pontificaux, viennent les actes épiscopaux qui semblent donc bien loin des premiers actes, ceux des comtes. Le premier acte d'un évêque est copié au folio 104. Il s'agit d'un acte de l'évêque de Châlons, autre sujet d'étonnement dans le cartulaire d'un établissement qui appartient au diocèse de Troyes et à la province ecclésiastique de Sens. Le premier acte d'un évêque de Troyes n'est d'ailleurs copié qu'au folio 106. Cette position correspond-elle à une relégation volontaire ? Il est vrai que le contexte de tensions entre le comte, fondateur de SaintÉtienne, et les évêques de Troyes est bien connu ${ }^{16}$. De même, les heurts entre la collégiale comtale et le pouvoir épiscopal ainsi que les rivalités de chapitre entre celui de Saint-Étienne et celui, cathédral, de SaintPierre, furent nombreux, comme en témoigne d'ailleurs le cartulaire qui reproduit les plaintes et les arbitrages rendus à propos de nombreuses

commission départementale des Monuments historiques du Pas-de-Calais, $2^{\mathrm{e}}$ série, 8 , 1970, p. 505-541 ; John S. Ott, Bishops, Authority and Community in Northwestern Europe, c. 1050-1150, Cambridge, Cambridge University Press, 2015.

15 Paris, BnF, ms. lat. 17098, fol. 31r-32r. Dans l'acte de première dotation du temporel, la collégiale est déjà fondée, et le bâtiment semble construit. On ignore donc la date précise de sa fondation.

${ }^{16}$ La dégradation des liens entre comtes de Champagne et évêques de Troyes culmine dans les années 1170, avec les tensions opposant le comte Henri le Libéral et l'évêque Mathieu, au sujet de l'exemption de la collégiale Saint-Étienne de Troyes, effective entre 1171-1172 et 1177. Voir Michel Bur, La Formation du comté de Champagne : v. 950 - v. 1150, Nancy, Université de Nancy II, 1977, notamment p. $72-81$ (à propos des difficiles rapports d'Henri le Libéral avec l'épiscopat), p. 181-189 et p. 496 (sur l'exemption de Saint-Étienne). 
querelles juridiques entre ces acteurs ${ }^{17}$. Faut-il voir dans l'organisation du cartulaire de Saint-Étienne un reflet du contexte politique et juridique de la Champagne méridionale du XIII ${ }^{\mathrm{e}}$ siècle ?

L'organisation du cartulaire de Saint-Étienne est surtout marquée par la division entre monde laïque et monde ecclésiastique. Comme le premier acte concernant l'établissement, celui de première dotation, émane d'un laïc, il semblait logique que les actes des laïcs soient transcrits en premier et que les actes émanant d'autorités religieuses le soient en second, sans pour autant que primeur signifie primauté ou prééminence. D'ailleurs, cette séparation entre monde laïque et monde ecclésiastique rappelle la division entre Liber pontificum et Liber principum, pour des cartulaires de chancellerie qui datent de $1271^{18}$, c'est-à-dire de quelques années avant la première campagne de cartularisation de Saint-Étienne. Il serait étonnant qu'il s'agisse là d'un hasard, puisque la collégiale séculière, qui jouxtait le palais des comtes à Troyes, remplissait des fonctions de chancellerie, conservant par exemple

\footnotetext{
${ }^{17}$ Les tensions entre l'évêque de Troyes et la collégiale Saint-Étienne concernent notamment les droits de justice que possède la communauté canoniale dans la capitale des comtes de Champagne et en particulier la justice du clos, bafouée à plusieurs reprises par les évêques (affaire Jean de Calais, 1298-1300; affaire Clément, années 1320). Les conflits entre la collégiale fondée par les comtes et le chapitre cathédral concernent quant à eux aussi bien des questions de préséance (place de Saint-Étienne dans les processions, circuits de celles-ci) que des affaires de concurrence dans la gestion des temporels respectifs des institutions, l'un des premiers contentieux entre les deux chapitres, en 1212, ayant d'ailleurs pour sujet la possession de deux ilôts sis devant le moulin du Pré, propriété de Saint-Pierre.

${ }^{18}$ Paris, BnF, ms. lat. 5993 A, Liber pontificum (1271), 55 fol. ; Paris, BnF, Cinq Cents de Colbert, vols. 56, 57 et 58, Liber principum (copie du XVII siècle d'un ms. datant de 1271). Autre copie du Liber principum: Paris, BnF, nouv acq. lat. 2454 (XVII ${ }^{\mathrm{e}}$ siècle). Dans le contexte de la minorité de Jeanne de Navarre et de la régence de Blanche d'Artois, assumée au nom de cette dernière par son second mari, Edmond de Lancastre, qui pourtant ne semble pas très intéressé par la Champagne, un cartulaire de chancellerie en deux volumes fut rédigé en 1272 : le premier volume (le Liber pontificum) contenait des actes émanant d'auteurs ecclésiastiques alors que le second (le Liber principum) était uniquement composé d'actes d'auteurs laïques. Voir Robert-Henri Bautier, "Cartulaires de chancellerie et recueils d'actes des autorités laïques et ecclésiastiques », dans Les Cartulaires, op. cit., p. 363-377 (notamment p. 368-369). 
les rôles des fiefs de Champagne ${ }^{19}$. Les chanoines de Saint-Étienne ontils participé à la rédaction du Liber pontificum et du Liber principum et en ont-ils reproduit les choix de classement, au moment de la création du cartulaire de leur propre institution? L'imitation des choix de classement serait donc une preuve de plus unissant la chancellerie comtale et le bureau d'écriture (pour ne pas dire scriptorium) de la collégiale SaintÉtienne de Troyes $^{20}$.

L'organisation des actes du cartulaire de Saint-Étienne de Troyes répond d'ailleurs à des dimensions pratiques qu'il ne faut pas occulter. Les actes des archidiacres, doyens de chrétienté et officiaux, très nombreux, y ont, par exemple, été copiés après les actes des collégiales, des abbayes et des prieurés, sans doute moins pour des raisons de préséance que pour une question de place.

Les processus de classement et d'organisation des actes renvoient à des attitudes cognitives, mécanismes d'acquisition des connaissances qui obéissent eux-mêmes à une logique et à des représentations. En partie construits par l'expérience, ils sont toujours ancrés dans un contexte de réalisation. Les classements des actes des cartulaires nous donnent donc à lire des hiérarchies qui ont pu être influencées par le contexte sociopolitique du temps et par le positionnement de l'institution commanditaire au sein de ce rapport de force. Certains phénomènes peuvent cependant s'expliquer par des causes plus pratiques : imitation d'une méthode expérimentée ailleurs et jugée efficace, besoin de place... Les causes politiques et pratiques peuvent d'ailleurs se cumuler. Je doute que les cartularistes de Saint-Étienne aient voulu marquer leur opposition

19 Theodore Evergates, The Chancery Archives of the Counts of Champagne: Codicology and History of the Cartulary-Register, Berkeley/Los Angeles/London, University of California Press, 1985.

${ }^{20}$ Il serait intéressant d'étudier l'organisation des actes à l'intérieur du Liber principum et du Liber pontificum, pour les comparer avec les hiérarchies des auteurs laïques et des auteurs ecclésiastiques dans le cartulaire de Saint-Étienne. 
à l'évêque de Troyes et lui manquer de respect en reléguant au folio 106 le premier de ses actes, le cartulaire n'étant pas destiné à une publicité externe à la collégiale et restant avant tout un objet pratique. Ils ne percevaient d'ailleurs sans doute pas la place des actes épiscopaux dans leur cartulaire comme le signe d'une relégation. S'ils s'en sont aperçus, ils n'en furent sans doute pas émus, étant donné les conflits avec le pouvoir épiscopal.

Que les différences dans le classement hiérarchique des actes des cartulaires de Saint-Étienne et de Saint-Loup répondent à des raisons politiques, pratiques ou à l'imitation de modèles différents, leur comparaison montre que, pour les comprendre, il faut avoir en tête le contexte de production de l'institution commanditaire.

\section{Cartulaires et intérêts des commanditaires : les mondes de Blanche et de Renier}

L'organisation des actes des cartulaires reflète parfois les intérêts particuliers, voire la personnalité, des commanditaires. La Champagne du $\mathrm{XIII}^{\mathrm{e}}$ siècle en fournit au moins deux autres exemples. Theodore Evergates rappelle en introduction de son édition du cartulaire dit de Blanche ${ }^{21}$, remis à la comtesse de Champagne au printemps de l'année 1225, que le manuscrit correspondait au cartulaire-registre $n^{\circ} 4$, suivant sa numérotation, cartulaire-registre datant des années 1222-1224 et qui compose les folios 1 à 207 d'un cartulaire de chancellerie issu de la réunion de plusieurs cartulaires-registres dans les années 1231-1232 $\left(\right.$ KK 1064) ${ }^{22}$. Blanche de Champagne (1180-1229), régente depuis la mort de son mari Thibaud III en 1201 et jusqu'à la majorité de son fils

\footnotetext{
${ }^{21}$ Paris, BnF, ms. lat. 5993 (1224), 181 fol. Pour une édition, voir The Cartulary of Countess Blanche of Champagne, éd. Theodore Evergates, Toronto/Buffalo/London, University of Toronto Press, 2009.

${ }^{22}$ Paris, AN, KK 1064 (1231-1232), 396 fog 24
} 
Thibaud IV en 1222, aurait demandé à avoir une copie du cartulaireregistre $n^{\circ} 4$, qui compte presque exclusivement des actes pris pendant ses années de régence. Elle aurait emmené ce cartulaire dans sa retraite de l'abbaye d'Argensolles. L'ordre des actes n'est pas le même entre les folios 1 à 207 du manuscrit KK 1064 et le cartulaire dit de Blanche. Par ailleurs, certains actes, et non des moindres, n'ont pas été recopiés dans le cartulaire destiné à la comtesse. Par exemple, le manuscrit KK 1064 s'ouvre par une série d'actes royaux de Philippe II, or ces derniers ne figurent pas dans le cartulaire dit de Blanche. Il en va de même pour la grande majorité des actes d'Érard de Brienne, présents au début du manuscrit KK 1064, mais absents du cartulaire de Blanche, comme si la comtesse avait voulu faire disparaître son rival dans la guerre civile des années 1210-1220, Érard de Brienne, et celui dont l'aide politique allait avec de nombreuses contraintes, Philippe II, dans ce qui est un réagencement personnel du matériau diplomatique des années de régence de Blanche. La comtesse n'a donc pas demandé une simple copie du cartulaire-registre $n^{\circ} 4$. Le reclassement des actes et l'élimination de certains documents ont permis de créer une image qui correspondait mieux aux intérêts et à la personnalité d'une comtesse qui voulait peutêtre garder près d'elle un miroir de sa régence, que ce soit pour des raisons mémorielles ou pour pouvoir justifier son action. Le cartulaire porte donc bien son surnom, puisqu'il nous donne à lire le monde selon Blanche.

Autre commanditaire, autre vision du monde dans le cartulaire du marchand Renier Acorre ${ }^{23}$, qui n'obéit pas aux mêmes logiques de

23 Paris, BnF, ms. fr. 8593 (seconde moitié du XIII ${ }^{\mathrm{e}}$ siècle), 187 fol. Pour une présentation du cartulaire de Renier Acorre, voir Thomas Lacomme, «Renier Acorre et la rue de Froidmantel : achats fonciers et distinction sociale (XIII ${ }^{\mathrm{e}}$ siècle) », dans Un quartier de la rue Saint-Thibault de Provins. Des jacobins et de Renier Acorre à 
classement que celui de la comtesse Blanche. L'organisation topographique des 546 actes du cartulaire n'est pas sans rappeler celle des biens fonciers du «plus riche et le plus important citoyen de Provins au $\mathrm{XIII}^{\mathrm{e}}$ siècle $^{24} »$. Son cartulaire était un objet pratique qu'il utilisait souvent: son organisation s'en ressent. Les actes les plus importants, ceux qui documentent la seigneurie banale qu'il s'est constituée dans la Brie, autour de l'actuel village de Gouaix, sont ainsi facilement consultables, puisqu'ils sont en tête du cartulaire. À la fin de celui-ci ont été copiés d'autres actes importants, ceux que les comtes de Champagne ont pris en faveur de Renier Acorre. Le choix de cet ordre perturbe la logique d'une organisation topographique pourtant dominante dans ce cartulaire et occasionne des doublons, parce que chacun de ces actes était déjà présent dans le cartulaire, classé là où le lieu principal du dispositif de l'acte l'exigeait. L'étude du dispositif des actes nous permet de dresser la liste des possessions du marchand et de les évaluer les unes par rapport aux autres, mais un autre élément nous permet déjà une première évaluation de ce patrimoine : l'organisation des actes, voulue par Renier Acorre lui-même et qui donc nous permet déjà de lire la façon dont il ordonnait son patrimoine, dans ce qui est un peu la trace du regard de Renier sur le monde d'Acorre.

Le rapprochement du cartulaire dit de Blanche et de celui commandé par Renier Acorre peut légitimement étonner, parce que ce sont des sources dont la nature même est différente : un cartulaire de chancellerie personnalisé par une comtesse et l'un des rares exemples de

l'Institution Sainte-Croix (XIII siècle-XXe siècle), Provins, Société d'Histoire et d'Archéologie de l'Arrondissement de Provins, 2017, p. 9-23, ici p. 10-12.

${ }^{24}$ Élisabeth Chapin, Les Villes de foire de Champagne des origines au début du $X I V^{e}$ siècle, Paris, Champion, 1937, p. 120. Voir aussi Pascale Verdier, «La constitution d'une seigneurie dans la Champagne du XIII ${ }^{\mathrm{e}}$ siècle: Renier Acorre, seigneur de Gouaix (1257-1289) », dans Seigneurs et seigneuries au Moyen Âge. Actes du $117^{e}$ Congrès national des sociétés savantes (Clermont-Ferrand, 1992), Paris, Éditions du CTHS, 1993, p. 99-110.226 
cartulaires privés laïques médiévaux que nous conservons aujourd'hui ${ }^{25}$. Le premier a été remis à Blanche en 1225, le second, moins précisément daté, a été réalisé dans la seconde moitié du XIII ${ }^{\mathrm{e}}$ siècle, ce qui fait qu'au moins 25 ans séparent les deux cartulaires et peut-être même jusqu'à 75 ans. La vision du monde dévoilée par les cartulaires commandés par ces deux personnalités ne peut donc pas être la même, puisqu'en un demisiècle la Champagne a connu des évolutions. Objectif dominant des deux cartulaires $^{26}$, échelle ${ }^{27}$, statut social des partenaires des actions juridiques qui y sont documentées ${ }^{28}$ : les différences sont si grandes qu'il aurait été difficile de les comparer. Un rapprochement est malgré tout possible, parce qu'ils permettent tous deux de comprendre comment, à travers l'organisation des actes, le commanditaire peut dévoiler sa vision du monde et la place qu'il occupe dans cet environnement sociopolitique reconstruit ou, du moins, celle qu'il prétend tenir. Les procédés sont différents d'un cartulaire à l'autre: l'élimination de certains auteurs d'actes dans le cartulaire dit de Blanche, la hiérarchisation entre les regroupements d'actes dans celui de Renier Acorre.

\footnotetext{
${ }^{25}$ Lucie Fossier et Olivier Guyotjeannin, « Cartulaires français laïques : seigneuries et particuliers », dans Les Cartulaires, op. cit., p. 379-410.

${ }^{26}$ La visée mémorielle semble dominante dans le cas du cartulaire dit de Blanche, alors que la visée pratique semble plus importante dans le cas de celui que fait réaliser Renier Acorre. La première visée n'exclut pourtant pas la seconde, et il ne serait pas pertinent de vouloir les opposer.

${ }^{27}$ Le cartulaire dit de Blanche, en raison de sa nature, embrasse l'ensemble du comté de Champagne, alors que celui que fait réaliser Renier Acorre, comme il correspond à la carte des possessions foncières du marchand italien, est centré sur la châtellenie de Provins.

${ }^{28}$ Difficile de comparer un seigneur de Gouaix avec une comtesse-régente de Champagne. Renier Acorre a occupé des fonctions importantes au sein de l'administration du comté puis à la cour du roi de France et il a pu ainsi rentrer en relations avec de puissantes personnes. Malgré cela, le résultat est que dans le cartulaire dit de Blanche apparaissent les principaux vassaux champenois et les grands établissements religieux du temps, alors que dans le cartulaire privé du marchand italien, ce sont tout au plus les principaux nobles et les grands établissements religieux de la région de Provins qui apparaissent.
} 
Les choix de classement des actes des cartulaires ne peuvent donc être pleinement compris que si le contexte de production de la source est connu, parce que les intérêts et même les personnalités des commanditaires les influencent. Les exemples tirés de la Champagne du XIII ${ }^{\mathrm{e}}$ siècle l'ont illustré, laissant à une synthèse, qui viendra peut-être un jour, le soin de le démontrer. Pour cela, il faut être en mesure de représenter non seulement les espaces géographiques, politiques et sociaux (re)construits par les cartulaires, mais aussi l'espace du cartulaire lui-même, où ces mondes prennent forme, parce que le classement des actes devient alors une grille de lecture de certaines des représentations des commanditaires. La méthode proposée ici, qui vise à modéliser graphiquement les cartulaires classés selon une logique hiérarchique, est donc un outil pour représenter l'espace codicologique et arriver à mieux comprendre non seulement les cartulaires, mais aussi les intérêts de leurs commanditaires. 